\title{
Exploration of 27 plasma immune markers: a cross-sectional comparison of 64 old psychiatric inpatients having unipolar major depression and 18 non-depressed old persons
}

Torfinn Lødøen Gaarden ${ }^{1 *}$ (D, Knut Engedal ${ }^{1,2}$, Jūrate Šaltytė Benth ${ }^{3,4}$, Marianne Larsen ${ }^{1}$, Bernhard Lorentzen ${ }^{1}$, Tom Eirik Mollnes ${ }^{5,6,7}$, Tor Magne Bjølseth ${ }^{1}$ and Albert Castellheim ${ }^{8}$

\begin{abstract}
Background: The prevalence of major depression (MD) according to population studies is the same for old (65 years and older) and younger adults. In contrast, an elevated proportion of old MD patients are hospitalized compared to younger adults with MD, indicating a need to expand the characteristics of old inpatients with MD. To illustrate this point, the association between inflammation and MD in old psychiatric inpatients is sparsely investigated even though an association between inflammation and treatment resistance among younger adults with MD has been reported. In this study, we aimed to explore the plasma concentrations of 27 immune markers in old inpatients with MD, and our purpose was to expand the understanding of inflammatory mechanisms in these patients.

Methods: Prior to electroconvulsive treatment of MD, we compared 64 inpatients with unipolar MD (mean age 75.2 years) and 18 non-depressed controls (mean age 78.0 years). Symptoms characterizing MD were assessed by the Hamilton Rating Scale of Depression (HRSD)-17, and the immune markers from peripheral blood plasma were analysed using multiplex assay technology. For statistical analysis of data, we used the independent samples median test, independent samples t-test, $x^{2}$-test, receiver operating characteristic curve analyses, stepwise discriminant analysis, and multivariate linear regression.

Results: Twenty-two immune markers representing pro- and anti-inflammatory, adaptive and trophic signalling had higher concentrations in the inpatients compared to the controls. Only the four immune markers IL-1 $\beta, I L-5, I L-10$ and IL-15 had concentrations below the lower detection limit in a considerable portion (above 20\%) of the patient cases. A combination of the concentration in plasma of TNF, vascular endothelial growth factor (VEGF), IL-1ß, IL-7 and monocyte chemotactic protein (MCP)-1, correctly classified 98.4\% of the depressed patients and $83.3 \%$ of the non-depressed controls. Plasma concentration of TNF and VEGF were associated with the HRSD-17 scores ( $p=0.017$ and 0.005 , respectively).
\end{abstract}

Conclusions: Our results indicate that several inflammatory mechanisms may be highly activated in old psychiatric inpatients with MD, and indicate that immune markers may contribute to a more comprehensive understanding of MD in old persons.

Trial registration: NCT01559324 ClinicalTrials.gov.

Keywords: Inflammation, Major depression, Cytokines, Immune markers, Ageing immune system, Old age

\footnotetext{
* Correspondence: Torfinnlodoen.gaarden@diakonsyk.no

${ }^{1}$ Department of Geriatric Psychiatry, Diakonhjemmet Hospital, Pastor Fangens

vei 18, 0854 Oslo, Norway

Full list of author information is available at the end of the article
}

(c) The Author(s). 2018 Open Access This article is distributed under the terms of the Creative Commons Attribution 4.0 International License (http://creativecommons.org/licenses/by/4.0/) which permits unrestricted use, distribution, and reproduction in any medium, provided you give appropriate credit to the original author(s) and the source, provide a link to the Creative Commons license, and indicate if changes were made. The Creative Commons Public Domain Dedication waiver (http://creativecommons.org/publicdomain/zero/1.0/) applies to the data made available in this article, unless otherwise stated. 


\section{Background}

According to population studies the prevalence of major depression (MD) in old adults (65 years and older) is reported to be about two to $5 \%$ in the USA, Germany and Norway [1-3]. Similarly, the prevalence of MD in the general population, including young and old adults, is reported to be three to $5 \%$ in Western Europe and Northern America [4]. Contrasting the stable prevalence of MD over time, the proportion of MD among psychiatric inpatients increases seven fold from below 30 years of age to age 70 and approaches $40 \%$ [5]. Consequently, departments of geriatric psychiatry in Norway dispose about one bed per 1000 aged 65 years and above, and non-responders to psychotherapy and anti-depressive medication treated in the primary health care are referred [6]. Thus, a better understanding of mechanisms involved in treatment resistant MD in old age may contribute to improve the quality of life and reduce the health care costs. As inflammation is associated with treatment resistance in younger adult patients with MD [7], exploring immune markers in old psychiatric inpatients with MD may contribute to expand the understanding of mechanisms that are involved.

The association between inflammation and MD in old psychiatric inpatients is sparsely examined. One study reported a $171 \%$ higher mean plasma level of interleukin (IL)- $1 \beta$ in 19 old inpatients compared to 21 non-depressed old controls [8]. In contrast, another study reported equal plasma concentration of IL-1 $\beta$, IL- 6 and tumour necrosis factor (TNF) between ten old MD psychiatric inpatients and ten non-depressed nursing home residents [9]. Additionally, several population-based, cross-sectional studies support an association between IL-6 and MD in old people [10-13] but the difference in plasma level of IL- 6 between the non-depressed and those with depressed mood seems modest [12]. Accordingly, a meta-analysis including mainly young adults found a medium effect size relationship between MD and the markers IL-1 and IL-6, but suggested a dose-response relationship between MD and inflammation [14].

Ageing is associated with increased inflammation [15] and together with age related cerebral hypo-perfusion inflammation is hypothesized to be involved in development of depression in old adults [16]. Accordingly, serotonin receptor $2 \mathrm{~B}(\mathrm{Htr} 2 \mathrm{~b})$ is reported to be upregulated during ageing in rodents [17] and Htr2b is reported to be co-localized with astrocytes and activated phagocytic microglia in peri-infarcted brain areas in humans [17]. The Astrocyte is the crucial microglia in the homeostasis of the human brain [18] and activated human astrocytes release cytokines initiating monocyte transmigration [19]. Thus, a connection between inflammation, microglia and mood regulation is suggested [20] and an understanding of these mechanisms may reveal useful biomarkers and new targets in treatment of MD in the old [20].

In our study, we decided to explore the plasma concentrations of 27 immune markers in old psychiatric inpatients with unipolar MD, because the association between inflammation and MD is sparsely investigated in old inpatients.

\section{Methods}

\section{The aims of the study}

In this study, we aimed to explore the plasma concentrations of 27 immune markers in old psychiatric inpatients with unipolar MD resistant to antidepressant treatment compared to a group of non-depressed old persons. Next, we aimed to ascertain whether a selection of plasma immune markers simultaneously might classify the depressed patients and the non-depressed controls. Finally, we aimed to explore the association between immune markers and the severity of symptoms characterizing MD in old persons.

\section{Study design}

The current study is a pre-electroconvulsive treatment, exploratory study comparing old psychiatric inpatients diagnosed with unipolar MD to a non-depressed control group of old persons. The study includes a subgroup of the patients from a larger randomized controlled trial [21], registered with the identifier: NCT01559324 at the online clinical database ClinicalTrials.gov.

\section{Inclusion criteria}

To be included, the patients were required to fulfil the criteria of the Diagnostic and Statistical Manual of Mental Disorders, Text Revision (DSM IV-TR) [22] of having a current episode of unipolar MD and having a score of at least 18 on the 17-item Hamilton Rating Scale for Depression (HRSD)-17 [23, 24]. The patient had to be between 60 to 85 years of age and had to be competent to give informed consent. All the patients were referred for electroconvulsive therapy (ECT).

\section{Exclusion criteria}

The following were exclusion criteria; bipolar depressive disorder, Parkinson's disease, schizophrenia, schizoaffective disorder, alcohol or substance abuse during the last three weeks, Mini Mental State Examination (MMSE) $[25,26]$ score of $<24$ or having a diagnosis of dementia. Patients with medical conditions contradicting ECT, including all acute medical conditions and life threatening medical conditions including an advanced stage of cancer, were excluded. Likewise, patients having received ECT within the previous six months were excluded. 


\section{Patients recruited}

We recruited Norwegian-speaking patients at the Diakonhjemmet Hospital, Department of Geriatric Psychiatry, a public hospital of Oslo, Norway. The department serves approximately 28,000 inhabitants aged 65 years and older. The patients were recruited during the period 1 September 2009 to 1 May 2013. Figure 1 depicts the flowchart of the recruitment process for the patients and controls. Ninety-seven patients were assessed for eligibility and 64 patients were included in the trial. Among the 33 excluded patients, 23 met exclusion criteria; two did not meet inclusion criteria; six withdrew their consent and two had their diagnosis altered.

\section{Diagnostic procedure}

Unipolar MD was diagnosed according to the DSM-IV-TR criteria [22]. Screening for psychiatric co-morbidity was performed by a consensus between two independent senior consultants in geriatric psychiatry after a standardized clinical interview including the diagnostic structural tool Mini-international Neuropsychiatric Interview (specifically the MINI-Plus) [27, 28]. The HRSD-17 was used to rate the severity of the symptoms characterizing MD.

\section{Physical examination}

All included patients underwent a routine physical examination with special attention to the cardiac-, respiratoryand nervous systems as a preparation for anaesthesia. The physical examination also included assessment for body mass index (BMI), blood sedimentation reaction (SR), C-reactive protein (CRP), leukocytes, haemoglobin, electrolytes, creatinine, liver-enzymes, thyroxine, HbA1c and glucose. Present and previous physical diseases affecting the immune system activity (e.g., cancer, inflammatory diseases and infections) were registered. We measured the cumulative medical burden of physical diseases using the Cumulative Illness Rating Scale for Geriatric Patients (CIRS-G) [29] (Table 1). One item regarding psychiatric disorders was excluded from the CIRS-G. The use of prescribed drugs was registered.

\section{Control group}

We recruited 20 non-depressed Norwegian speaking old adults from a community senior citizens centre as a control group (Table 1). Two of the controls were excluded because they had an advanced stage of cancer. This exclusion criterion also applied to the patient group. Symptoms of depression were assessed by the HRSD-17 (Table 1). We measured the cumulative medical burden of physical diseases using the CIRS-G (Table 1) from which the psychiatric item was excluded. Apart from assessing BMI, we did not perform physical examinations, and we did not register the use of psychotropic drugs in the controls.

\section{Blood sampling}

EDTA-plasma in peripheral blood was collected between 08.00 and $10.00 \mathrm{a}$. m. from the patients and between 10.00 and $11.00 \mathrm{a}$. m. from the controls. The blood was collected from patients before interventions with ECT. The blood was immediately centrifuged at $4{ }^{\circ} \mathrm{C}$ at $3000 \times \mathrm{g}$ for $15 \mathrm{~min}$ and immediately stored in a local bio-bank at $-80{ }^{\circ} \mathrm{C}$.

\section{Laboratory analysis}

The plasma samples were analysed (in a hospital laboratory in Bodø, Norway) using a multiplex cytokine assay (Bio-Plex Human Cytokine 27-Plex Panel; Bio-Rad

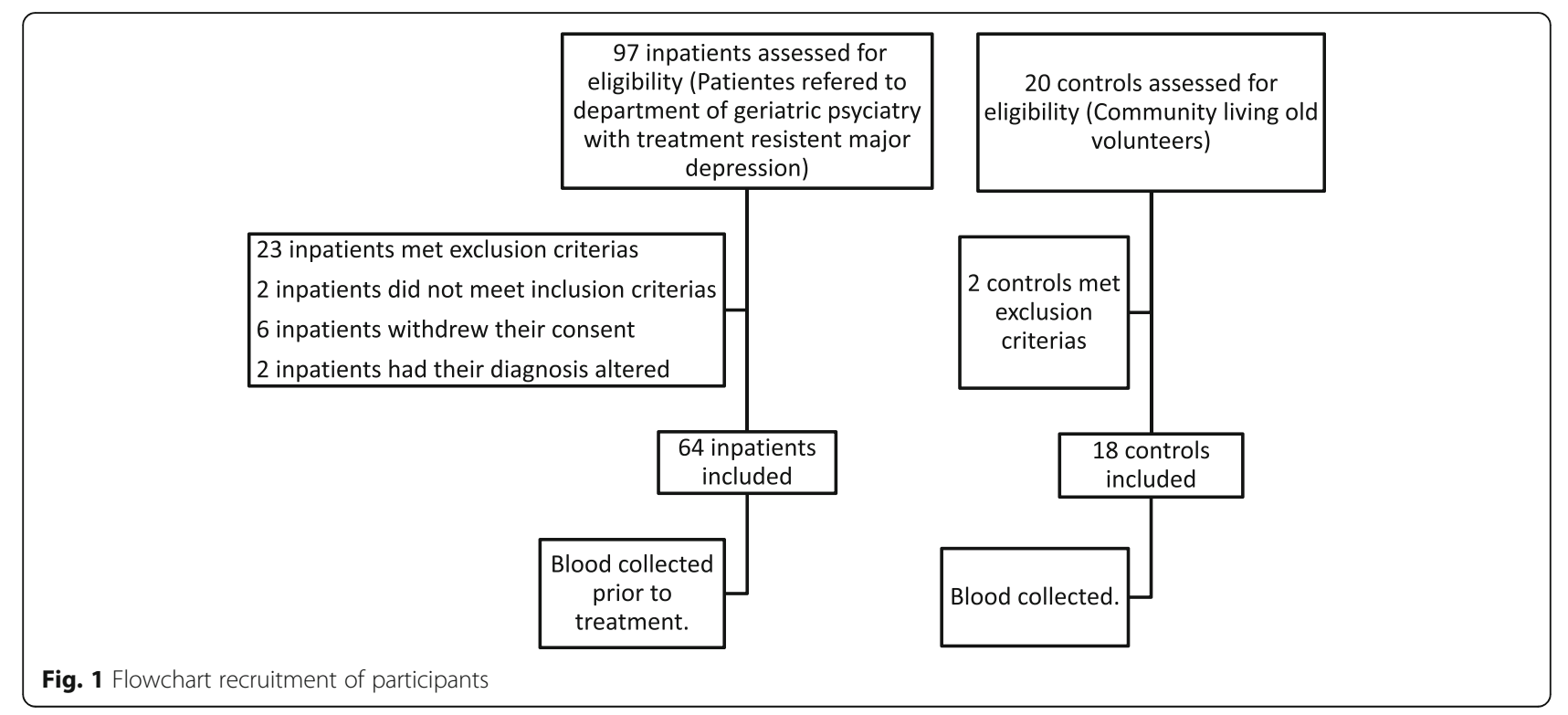


Table 1 Characteristics of the patient group and the nondepressed control group

\begin{tabular}{llll}
\hline & Patients, N=64 & Controls, N=18 \\
& Mean (SD), N (\%) & Mean (SD), N (\%) & $P$-value \\
\hline HRSD-17 & $23.1(4.6)$ & $2.7(2.7)$ & $<0.001^{\mathrm{a}}$ \\
MMSE & $27.7(1.8)$ & $28.2(2.1)$ & $0.432^{\mathrm{a}}$ \\
Age, years & $75.2(6.3)$ & $78.1(4.8)$ & $0.083^{\mathrm{a}}$ \\
Education, years & $13.6(3.0)$ & $13.4(2.8)$ & $0.805^{\mathrm{a}}$ \\
BMI & $23.3(4,6)$ & $24.2(4.1)$ & $0.422^{\mathrm{a}}$ \\
CIRS-G & $6.8(3.6)$ & $5.4(2.4)$ & $0.139^{\mathrm{a}}$ \\
Regular intake of & $5.2(2.3)$ & & \\
drugs, number & & & \\
Gender, female & $35(54.7 \%)$ & $12(66.7 \%)$ & $0.427^{\mathrm{b}}$ \\
& Median (Q1,Q3) & & \\
$\begin{array}{l}\text { Current depression } \\
\text { in weeks }\end{array}$ & $28(13 ; 77)$ & & \\
\hline
\end{tabular}

Abbreviations: CIRS-G cumulative illness rating scale for geriatric patients, HRSD-17 Hamilton rating scale of depression, MMSE Mini mental state examination, $N$ number, $S D$ standard deviation, $B M I$ body mass index andependent Samples $t$-test

${ }^{\mathrm{b}} \mathrm{X}^{2}$-test.

Laboratories Inc., Hercules, CA, USA) containing the following immune markers: IL-1 $\beta$, IL-1 receptor antagonist (ra), IL-2, IL-4, IL-5, IL-6, IL-7, IL-8, IL-9, IL-10, IL-12 (p70), IL-13, IL-15, IL-17, eotaxin, basic fibroblast growth factor (bFGF), granulocyte-colony stimulating factor (G-CSF), granulocyte macrophage colony stimulating factor (GM-CSF), interferon (IFN)- $\gamma$, interferon-inducible protein (IP)-10, monocyte chemotactic protein (MCP)-1, macrophage inflammatory protein (MIP)-1 $\alpha$, MIP-1 $\beta$, platelet derived growth factor (PDGF)-BB, regulated upon activation $\mathrm{T}$ cell expressed and secreted (RANTES), tumour necrosis factor (TNF)- $\alpha$ and vascular endothelial growth factor (VEGF) (Table 3). The samples were analysed on a Multiplex Analyzer (Bio-Rad Laboratories) according to instructions from the manufacturer. The lower detection limit was in the range of 0.24$18.84 \mathrm{pg} / \mathrm{ml}$ for the different immune markers. Values measured below the lower detection limit were extrapolated beyond standard range and values out of range were given the value $0.001 \mathrm{pg} / \mathrm{ml}$ in the statistical analysis. We classified the immune markers in the categories; proand anti-inflammatory, adaptive [30] and trophic based on their main signalling (Table 2).

\section{Statistical analysis}

The demographic and clinical characteristics were presented as group means and standard deviations (SDs) or frequencies and percentages. We compared group characteristics of patients and controls, as well as patients with and without physical diseases affecting the immune system activity, using independent samples $t$-test and $\chi^{2}$-test. The immune markers were non-normally distributed, and therefore we described them using medians and first and third quartiles. We conducted group comparisons between the patients with and without physical diseases affecting the immune system activity as well as between the patients and the controls using the independent samples median test. As the distribution of most immune markers was highly skewed, we used the LN-transformed values in all further analysis. We assessed correlations among immune markers using Pearson's correlation coefficients.

Receiver operating characteristic (ROC) curve analysis was performed on 27 immune markers to assess their ability to classify depressed patients and non-depressed controls. We selected the immune markers with an area under the ROC curve (AUC) of at least 0.85 and a specificity of at least 0.85 for a stepwise discriminant analysis (DA), as we aimed at defining a selection of a few factors that could simultaneously classify depressed patients and non-depressed controls. We adopted a cut-off of 0.85 to ensure that only immune markers with good classification ability would be included in the DA. The immune markers chosen by a stepwise DA were further explored in bivariate and multivariate linear regression models for a continuous HRSD-17 score. We entered the interaction terms between each immune marker and the variable identifying patients and controls into the regression model. A significant interaction implies that the immune marker is associated to the HRSD-17 score differently among the depressed patients and the non-depressed controls. All non-significant interactions were excluded. Finally, we adjusted the multivariate regression model for gender, age, CIRS-G scores and BMI.

The IBM SPSS Statistics 22 software and STATA v.12 were used for statistical analyses. All tests were two-sided, and we deemed the results with $p$-values below 0.05 to be statistically significant.

\section{Results}

Concentration of plasma immune markers in the patients versus the controls

We found higher concentrations of 22 immune markers (IL-1 $\beta$, IL-1ra, IL-2, IL-5, IL-6, IL-7, IL-8, IL-9, IL-12, IL-13, IL-15, IL-17, eotaxin, bFGF, G-CSF, GM-CSF, MCP-1, MIP-1 $\beta$, PDGF-BB, RANTES, TNF and VEGF) and lower concentration of IP-10 in the patients compared to the controls (Table 2). On the other hand, the concentrations of IL-4, IL-10, IFN- $\gamma$ and MIP- $1 \alpha$ were not significantly different between the two groups (Table 2).

Only four immune markers (IL-1 $\beta$, IL-5, IL-10 and IL-15) had concentrations below the lower detection limit in a considerable portion $(77,78,91$ and $39 \%$ respectively) of the patient cases. The remaining 23 immune markers had concentrations above the lover detection limits in $81-100 \%$ of the patient cases. In contrast, 18 immune markers (IL-1 $\beta$, IL-1ra, IL-2, IL-5, IL-6, IL-7, IL-9, IL-10, IL-12, IL-13, IL-15, 
Table 2 Descriptive statistics of the plasma immune markers

\begin{tabular}{|c|c|c|c|c|}
\hline \multicolumn{2}{|c|}{ Immune markers } & \multirow{2}{*}{$\begin{array}{l}\text { Patients, } N=64 \\
\text { Median }\left(\mathrm{Q}_{1}, \mathrm{Q}_{3}\right), \mathrm{pg} / \mathrm{ml}\end{array}$} & \multirow{2}{*}{$\begin{array}{l}\text { Controls, } N=18 \\
\text { Median }\left(\mathrm{Q}_{1}, \mathrm{Q}_{3}\right), \mathrm{pg} / \mathrm{ml}\end{array}$} & \multirow[b]{2}{*}{$P$-value ${ }^{f}$} \\
\hline Name & $\overline{\text { Signalling }}$ & & & \\
\hline IL-1 $\beta$ & Pro-infl. & $1.4(1.1 ; 2.0)^{a}$ & $0.5(0.3 ; 0.6)^{\mathrm{e}}$ & $<0.001$ \\
\hline IL-6 & Pro-infl. & $6.0(4.0 ; 8.0)$ & $2.0(0.9 ; 2.3)^{\mathrm{e}}$ & 0.001 \\
\hline TNF & Pro-infl. & $25(14 ; 38)$ & $10(3 ; 13)^{\mathrm{e}}$ & $<0.001$ \\
\hline $\mathrm{IL}-12$ & Pro-infl. & $9.0(6.0 ; 14.8)$ & $0.2(0.0 ; 7.5)^{e}$ & 0.030 \\
\hline IL-17 & Pro-infl. & $13(4 ; 27)$ & $0.0(0.0 ; 0.0)^{\mathrm{e}}$ & $<0.001$ \\
\hline IFN- $\gamma$ & Pro-infl. & $46(22 ; 77)$ & $25(6 ; 39)$ & 0.067 \\
\hline PDGFBB & Pro-infl. & $17(7 ; 37)$ & $2.0(0.0 ; 4.8)^{\mathrm{e}}$ & 0.003 \\
\hline IL-8 & Pro-infl. & $10(8 ; 13)$ & $5.0(3.8 ; 7.0)$ & $<0.001$ \\
\hline Eotaxin & Pro-infl. & $60(47 ; 84)$ & $30(25 ; 43)$ & 0.001 \\
\hline IP-10 & Pro-infl. & $574(424 ; 732)$ & $725(577 ;$ 1000) & 0.047 \\
\hline MCP-1 & Pro-infl. & $20(17 ; 26)$ & $12(9 ; 14)$ & $<0.001$ \\
\hline MIP-1a & Pro-infl. & $3.0(2.0 ; 4.0)$ & $2.0(0.9 ; 3.0)^{\mathrm{e}}$ & 0.052 \\
\hline MIP-1 $\beta$ & Pro-infl. & $54(46 ; 65)$ & $42(35 ; 52)$ & 0.022 \\
\hline RANTES & Pro-infl. & $1116(633 ; 2711)$ & $571(347 ; 850)$ & 0.016 \\
\hline IL-1ra & Anti-infl. & $67(42 ; 103)$ & $18(0 ; 29)^{\mathrm{e}}$ & 0.005 \\
\hline IL-10 & Anti-infl. & $0.0(0.0 ; 1.1)^{c}$ & $0.0(0.0 ; 0.6)^{\mathrm{e}}$ & 0.839 \\
\hline IL-2 & Adaptive & $5.0(2.3 ; 8.0)$ & $0.1(0.0 ; 2.3)^{\mathrm{e}}$ & 0.001 \\
\hline $\mid \mathrm{L}-4$ & Adaptive & $2.0(1.0 ; 2.0)$ & $1.0(1.8 ; 1.0)$ & 0.208 \\
\hline IL-5 & Adaptive & $1.2(1.0 ; 1.5)^{b}$ & $0.9(0.8 ; 1.1)^{\mathrm{e}}$ & $<0.005$ \\
\hline IL-7 & Adaptive & $6.0(4.3 ; 7.8)$ & $1.3(0.1 ; 2.3)^{\mathrm{e}}$ & $<0.001$ \\
\hline IL-9 & Adaptive & $12(8 ; 15)$ & $3.5(0.4 ; 8.3)^{\mathrm{e}}$ & 0.003 \\
\hline IL-13 & Adaptive & $4.5(3.0 ; 8.0)$ & $2.0(1.3 ; 3.0)^{\mathrm{e}}$ & 0.002 \\
\hline IL-15 & Adaptive & $2.5(0.0 ; 4.8)^{d}$ & $0.0(0.0 ; 0.0)^{\mathrm{e}}$ & $<0.001$ \\
\hline GMCSF & Trophic & $13(9 ; 18)$ & $6.0(0.7 ; 10.0)$ & $<0.001$ \\
\hline GCSF & Trophic & 25. $(16 ; 35)$ & $0.0(0.0 ; 12.3)^{e}$ & $<0.001$ \\
\hline bFGF & Trophic & $26(15 ; 36)$ & $0.0(0.0 ; 15.5)^{\mathrm{e}}$ & 0.001 \\
\hline VEGF & Trophic & $15(9 ; 23)$ & $0.0(0.0 ; 1.5)^{\mathrm{e}}$ & $<0.001$ \\
\hline
\end{tabular}

Abbreviations: Anti-infl. Anti-inflammatory, bFGF basic fibroblast growth factor, G-CSF granulocyte-colony stimulating factor, GM-CSF granulocyte macrophage colony stimulating factor, IFN interferon, IL interleukin, IP-10 interferon-inducible protein, MCP-1 monocyte chemotactic protein, MIP macrophage inflammatory protein, $\mathrm{ml}$ millilitre, $N$ number, PDGF-BB platelet derived growth factor-BB, $p g$ picogram, Pro-infl. Pro-inflammatory, $Q$ quartile, RANTES regulated upon activation $\mathrm{T}$ cell expressed and secreted, TNF tumour necrosis factor, VEGF vascular endothelial growth factor

a The concentration values were extrapolated beyond standard range in 49 cases.

b The concentration values were extrapolated beyond standard range in 50 cases.

c The concentration values were extrapolated beyond standard range in 23 cases and the concentration values were out of range in 35 cases.

${ }^{d}$ The concentration values were extrapolated beyond standard range in three cases and the concentration value were out of range in 22 cases.

e The concentration values were out of range or extrapolated beyond standard range in more than $20 \%$ of the cases.

${ }^{\mathrm{f}}$ Independent samples median test.

IL-17, bFGF, G-CSF, MIP-1 $\alpha$, PDGF-BB, TNF and VEGF) had concentrations below the lower detection limit in more than $20 \%$ of the cases in the controls (Table 2).

Gender, age, physical health, BMI, cognition and education were not significantly different between the groups (Table 1).

The impact of physical disorders on the immune markers within the patient group

Within the patient group, 37 patients with reported physical diseases affecting the immune activity had worse but not significantly different $(p=0.074)$ physical health as measured with CIRS_G (mean CIRS-G = 7.5) compared to 27 patients without reported physical diseases affecting the immune system activity (mean CIRS-G =5.9) (Additional file 1: Table S1). Of the 27 assessed immune markers, only the plasma concentration of IL-5, IL-8 and VEGF were significantly higher in the patients with reported physical diseases affecting the immune system activity $(p=0.015,0.020$ and 0.016 , respectively) compared to the patients without physical diseases affecting the immune system activity. Because 
of the similarity in concentration of immune markers between these two groups of patients, we treated the patients as one group.

\section{Classification of patients and controls by a panel of plasma immune markers}

According to the ROC curve analyses (Table 3), nine markers (IL-1 $\beta$, IL-1ra, IL-7, IL-8, IL-17, GM-CSF, MCP-1, TNF and VEGF) discriminated well between the patients and the controls as defined by the area under the ROC curve (AUC) of at least 0.85 , and with a specificity of at least 0.85 . The concentrations of all immune

Table 3 The receiver operating characteristic curve analyses of plasma immune markers

\begin{tabular}{|c|c|c|c|}
\hline Immune markers & $\mathrm{AUC}^{\mathrm{a}} \mathrm{Cl}(95 \%)$ & Sensitivity & Specificity \\
\hline $\mathrm{IL}-1 \beta$ & $0.97(0.93 ; 1.00)$ & 0.94 & 0.95 \\
\hline IL-1ra & $0.86(0.74 ; 0.99)$ & 0.89 & 0.85 \\
\hline $\mid \mathrm{L}-2$ & $0.84(0.72 ; 0.96)$ & 0.81 & 0.75 \\
\hline $\mid \mathrm{L}-4$ & $0.78(0.68 ; 0.87)$ & 0.63 & 0.90 \\
\hline IL-5 & $0.80(0.70 ; 0.90)$ & 0.81 & 0.75 \\
\hline IL-6 & $0.86(0.72 ; 1.00)$ & 0.94 & 0.80 \\
\hline IL-7 & $0.94(0.85 ; 1.00)$ & 0.94 & 0.90 \\
\hline $\mid \mathrm{L}-8$ & $0.93(0.86 ; 0.99)$ & 0.84 & 0.85 \\
\hline IL-9 & $0.86(0.74 ; 0.98)$ & 0.83 & 0.70 \\
\hline $\mid \mathrm{IL}-10$ & $0.52(0.38 ; 066)$ & 0.44 & 0.65 \\
\hline $\mid \mathrm{IL}-12$ & $0.84(0.70 ; 0.97)$ & 0.92 & 0.75 \\
\hline IL-13 & $0.81(0.69 ; 0.92)$ & 0.70 & 0.90 \\
\hline IL-15 & $0.81(0.74 ; 0.88)$ & 0.63 & 1.00 \\
\hline IL-17 & $0.89(0.79 ; 1.00)$ & 0.94 & 0.85 \\
\hline Eotaxin & $0.88(0.79 ; 0.97)$ & 0.80 & 0.80 \\
\hline bFGF & $0.86(0.77 ; 0.96)$ & 1.00 & 0.60 \\
\hline GCSF & $0.93(0.85 ; 1.00)$ & 0.98 & 0.75 \\
\hline GMCSF & $0.86(0.78 ; 0.95)$ & 0.56 & 1.00 \\
\hline $\mathrm{IFN}-\gamma$ & $0.71(0.58 ; 0.83)$ & 0.59 & 0.80 \\
\hline IP-10 & $0.69(0.56 ; 0.83)$ & 0.02 & 1.00 \\
\hline MCP-1 & $0.90(0.84 ; 0.97)$ & 0.81 & 0.90 \\
\hline MIP-1a & $0.72(0.58 ; 0.87)$ & 0.98 & 0.45 \\
\hline MIP-1 $\beta$ & $0.75(0.60 ; 0.89)$ & 0.78 & 0.65 \\
\hline PDGFBB & $0.83(0.70 ; 0.96)$ & 0.86 & 0.75 \\
\hline RANTES & $0.71(0.58 ; 0.84)$ & 0.64 & 0.75 \\
\hline TNF & $0.88(0.80 ; 0.96)$ & 0.75 & 0.90 \\
\hline VEGF & $0.98(0.95 ; 1.00)$ & 0.92 & 0.95 \\
\hline
\end{tabular}

Abbreviations: $A U C$ area under curve, $b F G F$ basic fibroblast growth factor, $C I$ confidence interval, G-CSF granulocyte-colony stimulating factor, GM-CSF granulocyte macrophage colony stimulating factor, IFN interferon, IL interleukin, IP-10 interferon-inducible protein, $M C P-1$ monocyte chemotactic protein, MIP macrophage inflammatory protein, PDGF-BB platelet derived growth factor-BB, RANTES regulated upon activation T cell expressed and secreted, TNF tumour necrosis factor, VEGF vascular endothelial growth factor

${ }^{a}$ AUC: Area under the receiver operating characteristic (ROC) curve markers correlated on a range of weakly to strongly (data not shown).

A step-wise discriminant analysis on the nine immune markers identified by ROC analysis suggested that the five markers, VEGF, IL-7, MCP-1, TNF and IL-1 $\beta$ (Fig. 2) are sufficient to classify the patients and the controls without loss of discriminatory power. The five markers correctly classify $98.4 \%$ of the patients and $83.3 \%$ of the controls, implying a correct classification rate of $95.1 \%$.

\section{The association between immune markers and the} severity of symptoms characterizing MD

No significant interactions were found in bivariate linear regression models, implying that there are no differences between the patients and the controls regarding the positive association between the HRSD-17 score and each of the five immune markers. In the multivariate linear regression model IL-7, TNF and VEGF were significantly associated with the HRSD-17 score. After adjusting for age, gender, CIRS-G and BMI; only TNF and VEGF remained significantly associated with the HRSD-17 score (Table 4). This model explained 53.3\% of the total variance of the HRSD-17 score.

\section{Discussion}

Effect size of the relationship between inflammation and MD Our finding showing a higher concentration of a variety of immune markers representing pro- and anti-inflammatory, adaptive and trophic signalling indicates that several immune mechanisms may be involved in MD in old psychiatric inpatients. Additionally, our results indicate that the immune system may be highly activated in old psychiatric inpatients with $\mathrm{MD}$, and this is consistent with a meta-analysis suggesting a dose-response relationship between inflammation and MD [14]. Further, the meta-analysis based mainly on adult outpatients reported medium effect size in the relationship between inflammation and MD [14]. The explorative approach of our study with a study population capable of detecting only large effect sizes between inflammation and MD, excluded reliable hypothesis testing. Therefore, identifying a more precise effect size in the relationship between inflammation and MD in old psychiatric inpatients requires further studies. However, our sample of old depressed inpatients is still the largest one in which study focused on the relationship between inflammation and MD. Thus, our study may contribute to better power calculations in the planning of future studies and testing of our hypothesis.

\section{Low plasma concentrations of immune markers}

We found low plasma concentrations of IL- $1 \beta$, IL-5, IL-10 and IL-15 in the patients and the controls, which is a finding in line with a previous study 


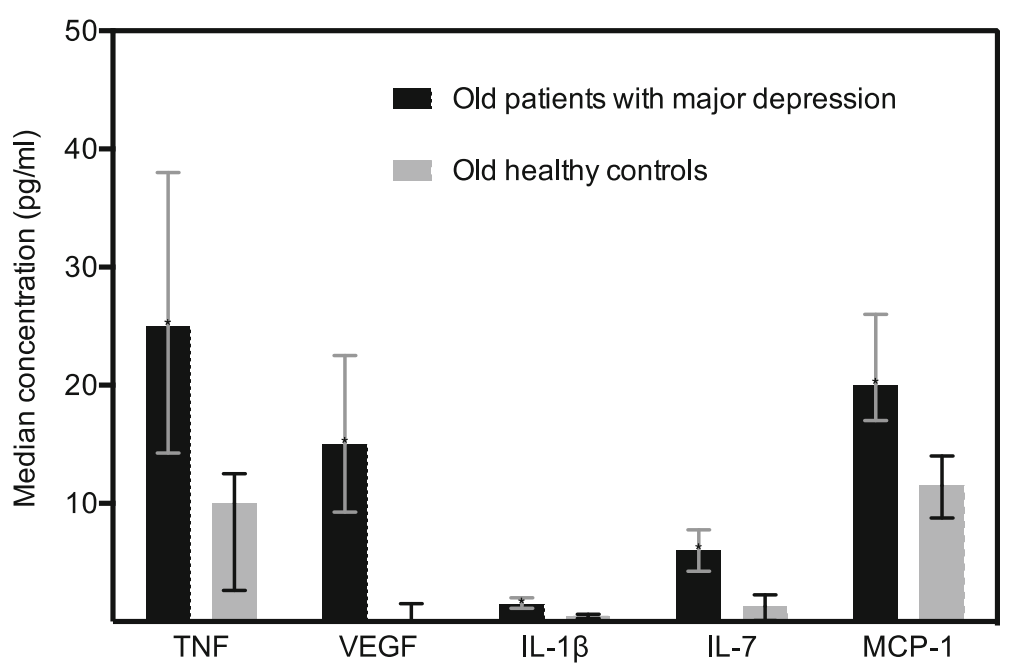

Fig. 2 Plasma immune markers associated with the severity of symptoms characterizing unipolar major depression. The median concentration of the plasma immune markers in old patients with unipolar major depression and old non-depressed controls are illustrated by the boxes. First and third quartiles are illustrated by the error bars. Abbreviations: pg/ml, picogram/millilitre; TNF, tumour necrosis factor; VEGF, vascular endothelial growth factor; IL, interleukin; MCP-1, monocyte chemotactic protein

reporting low serum concentration of IL-10 in adult depressed out-patients and non-depressed controls [31]. The low concentrations make our comparisons of these four immune markers between the two groups less reliable; further exploration of these markers in old psychiatric inpatients with MD may require more sensitive laboratory methods than the multiplex analyses [32]. Furthermore, 14 immune markers had plasma concentrations below the lower detection limit in more than $20 \%$ of the cases in the controls. However, values out of range were given the value $0.001 \mathrm{pg} / \mathrm{ml}$ and the concentrations were compared using medians to limit the risk of overestimating the differences between the groups.

\section{High levels of trophic cytokines in the patient group} compared to the controls

The trophic cytokines bFGF, G-CSF, GM-CSF and VEGF were higher in the patients compared to the controls. That is the opposite of what may be expected, as the more widely studied brain derived neurotrophic factor (BDNF) seems to be lower in untreated patients with MD compared to healthy controls [33]. On the other hand, higher level of VEGF in the patients with MD compared to the controls is a finding consistent with reports in previous studies [34]. Finally, pro-inflammatory cytokines like IL-1, TNF and INF- $\gamma$ may induce GM-CSF, and could at least explain the high concentration of GM-CSF [35].

\section{Classification of patients and controls by a selection of plasma immune markers}

Applying step-wise DA on a large number of immune markers implies some risk of detecting false effects and failing to select the best subset of markers able to describe differences between the two groups. To reduce the latter problem, we performed ROC curve analysis prior to DA to identify the most promising markers that could separate the patients from the controls.

Table 4 Linear regression models, immune markers associated with the Hamilton rating scale of depression

\begin{tabular}{|c|c|c|c|c|c|c|}
\hline \multirow[b]{3}{*}{ Immune markers } & \multicolumn{2}{|c|}{ Bivariate linear reg. Models } & \multicolumn{4}{|c|}{ Linear regression model assessing five immune markers simultaneously with HRSD-17 } \\
\hline & \multirow[b]{2}{*}{ Reg. coef. (SE) } & \multirow[b]{2}{*}{$p$-value } & \multicolumn{2}{|l|}{ Unadjusted } & \multicolumn{2}{|l|}{ Adjusted $^{a}$} \\
\hline & & & Reg. coef. (SE) & $p$-value & Reg. coef. (SE) & $p$-value \\
\hline$I L-1 \beta^{b}$ & $7.2(1.1)$ & $<0.001$ & $3.1(2.2)$ & 0.157 & $3.7(2.2)$ & 0.101 \\
\hline IL-7. & $3.2(0.5)$ & $<0.001$ & $1.7(0.7)$ & 0.017 & $1.5(0.7)$ & 0.059 \\
\hline MCP-1. ${ }^{b}$ & $8.9(1.9)$ & $<0.001$ & $2.4(1.9)$ & 0.212 & $3.0(2.0)$ & 0.146 \\
\hline TNF. $^{b}$ & $2.6(0.7)$ & $<0.001$ & $-2.5(1.0)$ & 0.015 & $-2.5(1.0)$ & 0.017 \\
\hline VEGF. & $1.8(0.2)$ & $<0.001$ & $1.2(0.4)$ & 0.002 & $1.1(0.4)$ & 0.005 \\
\hline
\end{tabular}

Abbreviations: HRSD-17 Hamilton rating scale of depression, CIRS-G cumulative illness rating scale for geriatric patients, BMI body mass index, Reg regression, coef coefficient, SE standard error, IL interleukin, MCP-1 monocyte chemotactic protein, TNF tumour necrosis factor, VEGF vascular endothelial growth factor

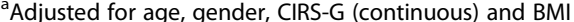

${ }^{\mathrm{b}}$ LN transformed. 
Our explorative statistical analyses elucidating immune markers that may classify old persons correctly into the depressed and the non-depressed group suggested that only five markers (VEGF, IL-7, MCP-1, TNF and IL-1 $\beta$ ) simultaneously classified about $95 \%$ of the persons correctly. To our knowledge, this has not been reported previously. However, the analyses imply a risk for Type 1 error and were not based on prior knowledge about the immune markers' ability to separate depressed patients from non-depressed controls. Therefore, the ability of these five immune markers to separate depressed from non-depressed should be replicated in a new population.

\section{The association between immune markers and the severity of symptoms characterizing MD}

We found a significant association between the severity of depression rated by the HRSD-17 and the levels of the three immune markers VEGF, IL-7, and TNF in the multivariate regression model. After adjusting the associations for age, gender and CIRS, only VEGF and TNF remained significantly associated to the HRSD-17 score. In contrast to the bivariate linear regression model, the multivariate regression model suggested a negative association between TNF and HRSD-17 (Table 4), which is likely due to over-adjustment in the model caused by strong correlations among the immune markers (Additional file 2: Table S2). Our finding deviates from that of Thomas et al. [8] who found a correlation between the level of IL- $1 \beta$ and the severity of the depression in old persons. Additionally, our finding contrasts with the results of Brambilla et al. [9], who found no correlation at all between the severity of the depression and the levels of inflammatory cytokines in old persons. However, Brambilla et al. [9] included only ten old patients with MD and consequently may not have had enough statistical power to detect putative group differences.

A small number of patients in this and previous studies, as well as differences in the study populations, may have contributed to the conflicting results.

\section{Limitations regarding the study groups}

Limited inclusion and exclusion criteria were defined for the controls in our study and did not ensure an optimal match of patients and controls. However, the groups did not differ significantly regarding gender, age, physical health, BMI, cognition and education, and this indicates an acceptable match between the groups. Still, we do not know if the groups differed in health behaviours, personality and emotional loneliness (Additional file 3: Table S3). Nevertheless, according to Mottus et al. [36] health behaviours such as smoking, alcohol intake and physical activity did not significantly affect inflammation in old. However, personality had a minor impact on inflammation in old persons [36]. Likewise, loneliness seems to have limited impact on inflammation in adults [37]. Thus, potential differences in health behaviour, personality and emotional loneliness between the patients and the controls in our study may potentially alter our results to a slight extent.

The controls were not examined in the same way as the patients. Physical diseases were assessed by self-reporting only; medication was not assessed, and MD was excluded by using the HRSD-17, not by a psychiatric interview. Consequently, we may have missed pro-inflammatory medical conditions and we may have included controls with MD in remission with ongoing anti-depressive medication leading to an underestimation of the differences between the groups. However, because physical diseases such as heart disease and cancer seem to have a small impact on the levels of immune markers [38-42] compared to $\mathrm{MD}$, a possible underestimation of the difference between the groups is probably small. This is also in line with our results where only three of 27 immune markers had significantly higher concentrations in patients with physical diseases affecting the immune system activity compared to the patients without. Likewise, if we accidentally included MD outpatients in remission and groups such as controls, this would probably not have altered our results because the levels of cytokines seem to be equal between outpatients treated with anti-depressive medication and healthy controls [43].

Our patient group represents old MD psychiatric inpatients that have not responded to medication and psychotherapy as outpatients, therefore our results should not be projected to younger adults or outpatients.

\section{Limitations in the study design and data collections}

The explorative cross-sectional design of this study excludes reliable testing of hypotheses and determination of the temporality in the associations demonstrated between immune markers and symptoms characterizing MD. Additionally, exploring a wide range of immune markers increases the risk of identifying false effects (Type I error) and the limited number of participants implies that only large effects sizes may be demonstrated (Type II error). Further, the accuracy of the data may be affected by the self-reporting nature of data sampling in the control group. Lastly, the plasma concentrations of several immune markers in the control group were below the lower-detection level, which may also influence the accuracy of comparison between the patient group and the controls.

\section{Conclusion}

Our results indicate that several inflammatory mechanisms may be highly activated in old psychiatric inpatients with MD, and indicate that immune markers 
may contribute to a more comprehensive understanding of MD in old persons.

\section{Additional files}

Additional file 1: Table S1. Characteristics of the patients. The variables; HRSD-17, age, BMI, CIRS-G, gender, number of drugs and current depression in weeks are compared between patients with and without physical diseases affecting the immune system activity. (DOCX $15 \mathrm{~kb}$ )

Additional file 2: Table S2. Correlation among the immune markers and the HRSD-17. Correlation among IL-1 $\beta, I L-7$, MCP-1, TNF, VEGF and the HRSD-17. (DOCX $17 \mathrm{~kb})$

Additional file 3: Table S3. Dataset supporting. Data on all characteristics and the concentrations of all 27 immune markers of 64 patients and 18 controls. (XLSX $25 \mathrm{~kb}$ )

\section{Abbreviations}

AUC: Area under curve; bFGF: Basic fibroblast growth factor; BMI: Body mass index; CIRS-G: Cumulative illness rating scale for geriatric patients; CRP: Creactive protein; DA: Discriminant analysis; ECT: Electroconvulsive therapy; GCSF: Granulocyte-colony stimulating factor; GM-CSF: Granulocyte macrophage colony stimulating factor; HRSD-17: Hamilton rating scale of depression; IFN: Interferon; IL: Interleukin; IP-10: Interferon-inducible protein; MCP-1: monocyte chemotactic protein; MD: Major depression; MIP: Macrophage inflammatory protein; ml: Millilitre; MMSE: Mini mental state examination; PDGF-BB: Platelet derived growth factor-BB; pg: Picogram; PIC: Pro-inflammatory cytokine; RANTES: Regulated upon activation T cell expressed and secreted; ROC: Receiver operating characteristic; SD: Standard deviation; SR: Sedimentation reaction; TNF: Tumour necrosis factor; VEGF: Vascular endothelial growth factor

\section{Acknowledgements}

Gro Strømnes Dybedal PsyD, PhD has been an important motivator in discussions involving interpretation of the data, and she conducted a major part of the assessments of the symptom severity. We thank Judith Krey Ludviksen for excellent technical performance of the cytokine analyses.

\section{Funding}

The collection of data in this study was sponsored by the Department of Geriatric Psychiatry, Diakonhjemmet Hospital.

All laboratory analyses were sponsored and done at the Research Laboratory, Nordland Hospital Bodø and K.G. Jebsen TREC, University of Tromsø. The article was written with a PhD grant, project number 2014113, from the South-Eastern Norway Regional Health Authority.

The Department of Geriatric Psychiatry and the Research Laboratory have together designed the study; they have both been involved in the interpretation of the data and in writing of the manuscript. The Department of Geriatric Psychiatry did collect all the data whereas the Research Laboratory did all the laboratory analysis. The PhD grant from the SouthEastern Norway Regional Health Authority was given when all the data was collected and analysed. The South-Eastern Norway Regional Health Authority has not contributed in study design, data collection, data interpretation or writing the manuscript.

\section{Availability of data and materials}

All data analysed during this study are included in this published article and its supplementary information files.

\section{Name of the institution at which the research was conducted}

Diakonhjemmet hospital, Department of Geriatric Psychiatry.

Pastor Fangens vei 18, 0854 Oslo, Norway.

\section{Authors' contributions}

TLG participated in recruiting patients and was a major contributor in writing the manuscript. KE participated in the planning of the study and was a major contributor in writing the manuscript. TMB participated in planning the study and recruiting patients and was a contributor in writing the manuscript. JŠB performed the statistical analyses and contributed in writing the manuscript. ML participated in recruiting patients and was a contributor in writing the manuscript. BL participated in the planning of the study and was a contributor in writing the manuscript. TEM participated in the planning of the study, interpretation of the data and was a contributor in writing the manuscript. AC participated in the planning of the study and was a major contributor in writing the manuscript. All authors have read and approved the final manuscript.

\section{Ethics approval and consent to participate}

The Regional South East Committee for Medical and Health Research Ethics in Norway has approved the study. Inclusions have been based strictly upon informed consent and the patient's signature.

\section{Consent for publication}

Not applicable.

\section{Competing interests}

The authors declare that they have no competing interests.

\section{Publisher's Note}

Springer Nature remains neutral with regard to jurisdictional claims in published maps and institutional affiliations.

\section{Author details}

'Department of Geriatric Psychiatry, Diakonhjemmet Hospital, Pastor Fangens vei 18, 0854 Oslo, Norway. ${ }^{2}$ Norwegian Centre of Ageing and Health, and the Department of Old Age Psychiatry, Oslo, Norway. ${ }^{3}$ Institute of Clinical Medicine, Campus Ahus, University of Oslo, Oslo, Norway. ${ }^{4}$ Health Services Research Unit, Akershus University Hospital, Lørenskog, Norway. ${ }^{5}$ Department of Immunology, Oslo University Hospital and K.G. Jebsen IRC, University of Oslo, Oslo, Norway. ${ }^{6}$ Research Laboratory, Nordland Hospital Bodø and K.G. Jebsen TREC, University of Tromsø, Tromsø, Norway. ${ }^{7}$ Centre of Molecular Inflammation Research, Norwegian University of Science and Technology, Trondheim, Norway. ${ }^{8}$ Department of Anaesthesiology and Intensive Care, Institute of Clinical Sciences, Sahlgrenska Academy, University of Gothenburg, Gothenburg, Sweden.

Received: 15 September 2017 Accepted: 12 June 2018

Published online: 25 June 2018

\section{References}

1. Kessler RC, Birnbaum H, Bromet E, Hwang I, Sampson N, Shahly V. Age differences in major depression: results from the National Comorbidity Survey Replication (NCS-R). Psychol Med. 2010;40(2):225-37.

2. Glaesmer H, Riedel-Heller S, Braehler E, Spangenberg L, Luppa M. Age- and gender-specific prevalence and risk factors for depressive symptoms in the elderly: a population-based study. Int Psychogeriatr. 2011;23(8):1294-300.

3. Sandanger I, Nygard JF, Sorensen T, Dalgard OS. Return of depressed men: changes in distribution of depression and symptom cases in Norway between 1990 and 2001. J Affect Disord. 2007;100(1-3):153-62.

4. Ferrari AJ, Somerville AJ, Baxter AJ, Norman R, Patten SB, Vos T, Whiteford HA. Global variation in the prevalence and incidence of major depressive disorder: a systematic review of the epidemiological literature. Psychol Med. 2013:43(3):471-81

5. Wetterling T, Junghanns K. Affective disorders in older inpatients. Int J Geriatr Psychiatry. 2004;19(5):487-92.

6. Borza T, Engedal K, Bergh S, Benth JS, Selbaek G. The course of depression in late life as measured by the Montgomery and Asberg depression rating scale in an observational study of hospitalized patients. BMC Psychiatry. 2015;15:191

7. Strawbridge R, Arnone D, Danese A, Papadopoulos A, Herane Vives A, Cleare AJ. Inflammation and clinical response to treatment in depression: a meta-analysis. Eur Neuropsychopharmacol. 2015;25(10):1532-43.

8. Thomas AJ, Davis S, Morris C, Jackson E, Harrison R, O'Brien JT. Increase in interleukin-1 beta in late-life depression. Am J Psychiatry. 2005;162(1):175-7.

9. Brambilla F, Maggioni M. Blood levels of cytokines in elderly patients with major depressive disorder. Acta Psychiatr Scand. 1998;97(4):309-13.

10. Bremmer MA, Beekman AT, Deeg DJ, Penninx BW, Dik MG, Hack CE, Hoogendijk WJ. Inflammatory markers in late-life depression: results from a population-based study. J Affect Disord. 2008;106(3):249-55. 
11. Tiemeier H, Hofman A, van Tuijl HR, Kiliaan AJ, Meijer J, Breteler MM. Inflammatory proteins and depression in the elderly. Epidemiology. 2003; 14(1):103-7.

12. Penninx BW, Kritchevsky SB, Yaffe K, Newman AB, Simonsick EM, Rubin S, Ferrucci L, Harris T, Pahor M. Inflammatory markers and depressed mood in older persons: results from the health, aging and body composition study. Biol Psychiatry. 2003;54(5):566-72.

13. Dentino AN, Pieper CF, Rao MK, Currie MS, Harris T, Blazer DG, Cohen HJ. Association of interleukin- 6 and other biologic variables with depression in older people living in the community. J Am Geriatr Soc. 1999;47(1):6-11.

14. Howren MB, Lamkin DM, Suls J. Associations of depression with C-reactive protein, IL-1, and IL-6: a meta-analysis. Psychosom Med. 2009;71(2):171-86.

15. Sandu RE, Buga AM, Uzoni A, Petcu EB, Popa-Wagner A. Neuroinflammation and comorbidities are frequently ignored factors in CNS pathology. Neural Regen Res. 2015;10(9):1349-55.

16. Taylor WD, Aizenstein HJ, Alexopoulos GS. The vascular depression hypothesis: mechanisms linking vascular disease with depression. Mol Psychiatry. 2013;18(9):963-74.

17. Buga AM, Ciobanu O, Badescu GM, Bogdan C, Weston R, Slevin M, Di Napoli M, Popa-Wagner A. Up-regulation of serotonin receptor $2 B$ mRNA and protein in the peri-infarcted area of aged rats and stroke patients. Oncotarget. 2016;7(14):17415-30.

18. Rajkowska G, Stockmeier CA. Astrocyte pathology in major depressive disorder: insights from human postmortem brain tissue. Curr Drug Targets. 2013;14(11):1225-36

19. Bethel-Brown $\mathrm{C}, \mathrm{Yao} \mathrm{H}, \mathrm{Hu} \mathrm{G}$, Buch S. Platelet-derived growth factor (PDGF)BB-mediated induction of monocyte chemoattractant protein 1 in human astrocytes: implications for HIV-associated neuroinflammation. J Neuroinflammation. 2012;9:262

20. Popa-Wagner A, Buga AM, Tica AA, Albu CV. Perfusion deficits, inflammation and aging precipitate depressive behaviour. Biogerontology. 2014;15(5):439-48

21. Bjolseth TM, Engedal K, Benth JS, Dybedal GS, Gaarden TL, Tanum L. Clinical efficacy of formula-based bifrontal versus right unilateral electroconvulsive therapy $(E C T)$ in the treatment of major depression among elderly patients: a pragmatic, randomized, assessor-blinded, controlled trial. J Affect Disord. 2015;175:8-17.

22. American Psychiatric Association. Diagnostic and Statistical Manual of Mental Disorders, Text Revision (DSM-IV-TR) fourth ed. edn. Washington: American Psychiatric Association; 2000.

23. Hamilton. A rating scale for depression. J Neurol Neurosurg Psychiatry. 1960; 23:56-62.

24. Williams JB. A structured interview guide for the Hamilton Depression Rating Scale. Arch Gen Psychiatry. 1988;45(8):742-7.

25. Engedal $K$, Haugen $P$, Gilje K, Laake P. Efficacy of short mental tests in the detection of mental impairment in old age. Compr Gerontol A. 1988;2(2):87-93.

26. Folstein MF, Folstein SE, McHugh PR. "Mini-mental state". A practical method for grading the cognitive state of patients for the clinician. J Psychiatr Res. 1975;12(3):189-98.

27. Mordal J, Gundersen O, Bramness JG. Norwegian version of the Miniinternational neuropsychiatric interview: feasibility, acceptability and test-retest reliability in an acute psychiatric ward. Eur Psychiatry. 2010;25(3):172-7.

28. Sheehan DV, Lecrubier $Y$, Sheehan KH, Amorim P, Janavs J, Weiller $E_{4}$ Hergueta T, Baker R, Dunbar GC. The Mini-International Neuropsychiatric Interview (M.I.N.I.): the development and validation of a structured diagnostic psychiatric interview for DSM-IV and ICD-10. J Clin Psychiatry. 1998;59(Suppl 20):22-33. quiz 34-57

29. Miller MD, Paradis CF, Houck PR, Mazumdar S, Stack JA, Rifai AH, Mulsant B, Reynolds CF 3rd. Rating chronic medical illness burden in geropsychiatric practice and research: application of the cumulative illness rating scale. Psychiatry Res. 1992;41(3):237-48.

30. Turner MD, Nedjai B, Hurst T, Pennington DJ. Cytokines and chemokines: at the crossroads of cell signalling and inflammatory disease. Biochim Biophys Acta. 2014;1843(11):2563-82.

31. Dhabhar FS, Burke HM, Epel ES, Mellon SH, Rosser R, Reus VI, Wolkowitz OM. Low serum IL-10 concentrations and loss of regulatory association between IL-6 and IL-10 in adults with major depression. J Psychiatr Res. 2009;43(11): 962-9.

32. Dossus L, Becker S, Achaintre D, Kaaks R, Rinaldi S. Validity of multiplexbased assays for cytokine measurements in serum and plasma from "non-diseased" subjects: comparison with ELISA. J Immunol Methods. 2009;350(1-2):125-32.
33. Kreinin A, Lisson S, Nesher E, Schneider J, Bergman J, Farhat K, Farah J, Lejbkowicz F, Yadid G, Raskin L, et al. Blood BDNF level is gender specific in severe depression. PLoS One. 2015;10(5):e0127643.

34. Clark-Raymond A, Halaris A. VEGF and depression: a comprehensive assessment of clinical data. J Psychiatr Res. 2013;47(8):1080-7.

35. Bhattacharya P, Budnick I, Singh M, Thiruppathi M, Alharshawi K, Elshabrawy H, Holterman MJ, Prabhakar BS. Dual role of GM-CSF as a pro-inflammatory and a regulatory cytokine: implications for immune therapy. J Interf Cytokine Res. 2015;35(8):585-99.

36. Mottus R, Luciano M, Starr JM, Pollard MC, Deary IJ. Personality traits and inflammation in men and women in their early 70s: the Lothian birth cohort 1936 study of healthy aging. Psychosom Med. 2013;75(1):11-9.

37. Mezuk B, Choi M, DeSantis AS, Rapp SR, Diez Roux AV, Seeman T. Loneliness, depression, and inflammation: evidence from the multi-ethnic study of atherosclerosis. PLoS One. 2016:11(7):e0158056.

38. Oliveira Miranda D, Soares de Lima TA, Ribeiro Azevedo L, Feres O, Ribeiro da Rocha JJ, Pereira-da-Silva G. Proinflammatory cytokines correlate with depression and anxiety in colorectal cancer patients. Biomed Res Int. 2014 2014:739650.

39. Jehn CF, Kuehnhardt D, Bartholomae A, Pfeiffer S, Krebs M, Regierer AC, Schmid P, Possinger K, Flath BC. Biomarkers of depression in cancer patients. Cancer. 2006:107(11):2723-9.

40. Musselman DL, Miller AH, Porter MR, Manatunga A, Gao F, Penna S, Pearce BD, Landry J, Glover S, McDaniel JS, et al. Higher than normal plasma interleukin-6 concentrations in cancer patients with depression: preliminary findings. Am J Psychiatry. 2001;158(8):1252-7.

41. Xiong GL, Prybol K, Boyle SH, Hall R, Streilein RD, Steffens DC, Krishnan R, Rogers JG, O'Connor CM, Jiang W. Inflammation markers and major depressive disorder in patients with chronic heart failure: results from the sertraline against depression and heart disease in chronic heart failure study. Psychosom Med. 2015;77(7):808-15.

42. Nikkheslat N, Zunszain PA, Horowitz MA, Barbosa IG, Parker JA, Myint AM, Schwarz MJ, Tylee AT, Carvalho LA, Pariante CM. Insufficient glucocorticoid signaling and elevated inflammation in coronary heart disease patients with comorbid depression. Brain Behav Immun. 2015:48:8-18.

43. Eller T, Vasar V, Shlik J, Maron E. Pro-inflammatory cytokines and treatment response to escitalopram in major depressive disorder. Prog NeuroPsychopharmacol Biol Psychiatry. 2008:32(2):445-50.

\section{Ready to submit your research? Choose BMC and benefit from:}

- fast, convenient online submission

- thorough peer review by experienced researchers in your field

- rapid publication on acceptance

- support for research data, including large and complex data types

- gold Open Access which fosters wider collaboration and increased citations

- maximum visibility for your research: over $100 \mathrm{M}$ website views per year

At BMC, research is always in progress.

Learn more biomedcentral.com/submissions 